\title{
Estado nutricional e sua associação com o perfil sintomatológico de pacientes em cuidados paliativos internados em um hospital de urgências do nordeste brasileiro
}

\author{
Nutritional status and its association with the symptomatological profile of patients in palliative \\ care admitted to an emergency hospital in Northeastern Brazil
}

DOI: $10.37111 /$ braspenj.2020352006

Rebeca Rocha Almeida

Larissa Monteiro Costa ${ }^{2}$

Fabiana Ferreira da Paixão Santos ${ }^{3}$

Jicelia Santos de Oliveira ${ }^{4}$

Joana Cardoso dos Santos

Victor Batista Oliveira ${ }^{6}$

\section{RESUMO}

Introdução: Os cuidados paliativos são prestados a pacientes com doenças irreversíveis, mediante a impossibilidade terapêutica de cura. $O$ tratamento paliativo se destina a amenizar os sintomas que acometem esses indivíduos, retratando a morte como algo natural e aceitável. Portanto, uma vez que são escassos os trabalhos que abordam essa temática, o presente estudo teve como objetivo avaliar os sintomas de pacientes em cuidados paliativos em um hospital de urgência do nordeste brasileiro e sua associação com o estado nutricional. Método: Foi realizado um estudo transversal analítico com amostragem por conveniência, com pacientes em cuidados paliativos internados no Hospital de Urgência de Sergipe, Aracaju-SE, durante o período de agosto a setembro de 2018. Foram aplicados os questionários Memorial Symptom Assessment Scale, para avaliação dos sintomas e a Avaliação Subjetiva Global Produzida Pelo Paciente, para o diagnóstico nutricional. Resultados: A amostra foi composta por 52 indivíduos, com média de idade de $59,62 \pm 15,51$ anos, a maior parte dos indivíduos era do sexo masculino $(53,85 \%)$, apresentava neoplasias e cerca de $41,86 \%$ estavam moderadamente desnutridos. Entre os sintomas avaliados, os mais frequentes foram: eu não pareço mais eu mesmo $(78,85 \%)$, perda de peso $(73,07 \%)$, dor $(69,23 \%)$, tristeza $(65,38 \%)$, boca seca $(57,69 \%)$, falta de energia $(55,76 \%)$. Foi observada uma associação, estaticamente significativa, entre os sintomas físicos e a escala geral com o estado nutricional dos pacientes $(p<0,05)$. Conclusão: Diante do exposto, foi possível concluir que a progressão da doença terminal envolve sintomas físicos, psicológicos, espirituais e sociais, os quais resultam da progressão da doença primária, assim como do seu tratamento e comorbidades, com direta associação ao estado nutricional.

\section{ABSTRACT}

Introduction: Palliative care are provided to patients with irreversible diseases, due to the therapeutic impossibility of cure. Palliative treatment is intended to alleviate the symptoms that affect these individuals, portraying death as something natural and acceptable. Therefore, since there are few studies addressing this theme, the present article aimed to assess the symptoms of patients in palliative care in an emergency hospital in northeastern Brazil and its association with nutritional status. Methods: An analytical cross-sectional study was carried out with a convenience sample of 52 individuals diagnosed with palliative care admitted to the Sergipe Emergency Hospital, from August to September 2018, the Memorial Symptom Assessment Scale questionnaires were applied for evaluation of symptoms, and the Subjective Global Assessment Produced by the Patient, for the nutritional diagnosis. Results: The sample consisted of 52 individuals with an average age of $59.62 \pm 15.51$ years, most of the individuals were male $(53.85 \%)$, had neoplasms and about $41.86 \%$ were moderately malnourished. Among the symptoms evaluated, the most frequent were: I don't look like myself anymore $(78.85 \%)$, weight loss $(73.07 \%)$, pain $(69.23 \%)$, sadness $(65.38 \%)$, mouth drought $(57.69 \%)$, lack of energy (55.76\%). A statistically significant association was observed between physical symptoms and the general scale with the patients' nutritional status ( $p<0.05)$. Conclusion: Given the above, it was possible to conclude that the progression of terminal illness involves physical, psychological, spiritual and social symptoms, which result from the progression of the primary disease, as well as its treatment and comorbidities, with a direct association with nutritional status.

\section{Aceito para publicação} $22 / 06 / 2020$

1. Nutricionista, Mestrado em Ciências da Saúde pela Universidade de Sergipe, Aracaju, Sergipe, Brasil.

2. Nutricionista, Mestre em Educação Física-UFS, Especialista em Nutrição da Saúde do Adulto e Idoso HU-UFS, Docente da Faculdade Estácio de Sergipe, Aracaju, SE, Brasil.

3. Nutricionista Graduada pela Faculdade Estácio de Sergipe - FASE, Aracaju, SE, Brasil.

4. icelia Santos de Oliveira:Nutricionista Graduada pela Faculdade Estácio de Sergipe - FASE, Aracaju, SE, Brasil.

5. Nutricionista Graduada pela Faculdade Estácio de Sergipe- FASE, Aracaju, SE, Brasil.

6. Nutricionista, Pós-Graduando no programa de mestrado de ciências da saúde, Pós-graduando em terapia nutricional enteral e parenteral, Aracaju, SE, Brasil. 


\section{INTRODUÇÃO}

Os cuidados paliativos (CP) são prestados a pacientes com doenças irreversíveis, mediante a impossibilidade terapêutica de cura, direciona-se ao controle dos sintomas e à melhor perspectiva de vida dos indivíduos, sem retardar ou apressar a morte, respeitando o processo natural da vida ${ }^{1,2}$.

Anualmente, cerca de 40 milhões de indivíduos necessitam de cuidados paliativos no mundo. No entanto, apenas $14 \%$ dessas pessoas o recebem a tempo e de forma adequada ${ }^{3}$. Segundo o The Quality of Death Index, que destaca a qualidade de morte entre os países ao redor do mundo, o Brasil encontra-se no $42^{\circ}$ lugar, no final da lista, próximo a países como Hungria, México, Tailândia e Venezuela ${ }^{4}$.

manejo dos sintomas encontrados em tais pacientes é essencial para o bem-estar desta população. Dentre os sintomas, cansaço, anorexia, ansiedade, sonolência, depressão, constipação, disfagia, dispneia e fraqueza são os mais prevalentes. Além disso, comprometem o estado nutricional e a qualidade de vida dos pacientes ${ }^{5}$.

A desnutrição nos pacientes, com o quadro clínico anteriormente exposto, pode estar associada à diminuição da ingestão alimentar e à inflamação sistêmica ${ }^{6}$. Esse quadro é responsável pelo risco de readmissões por alterações fisiológicas que influenciam negativamente o tratamento, sendo considerado um fator de risco para o aumento da morbimortalidade ${ }^{7}$. Apesar da alta incidência em ambiente hospitalar, a desnutrição é negligenciada e, dessa forma, não tratada ${ }^{8}$.

Mediante a escassez de trabalhos abordando a temática aqui discutida, o presente estudo teve como objetivo avaliar os sintomas de pacientes em cuidados paliativos, em um hospital de urgência do nordeste brasileiro, e sua associação com o estado nutricional.

\section{MÉTODO}

Realizou-se um estudo transversal analítico, com uma amostra por conveniência, composta por indivíduos com diagnóstico terapêutico de cuidados paliativos, internados no Hospital de Urgência de Sergipe, durante o período de agosto a setembro de 2018.

A amostra foi composta por indivíduos com idade superior a 18 anos, de ambos os sexos, e com diagnóstico atual de cuidados paliativos descritos pelo médico em prontuário clínico. Foram excluídos da amostra pacientes em coma e/ ou internados na unidade de terapia intensiva (UTI), com precaução de contato e/ou isolamento.

Foi utilizada a Avaliação Subjetiva Global Produzida pelo Próprio Paciente (ASG-PPP), adaptada por Gonzalez et al. ${ }^{2}$, classificando-os em: A, para bem nutrido; B, para moderadamente desnutrido ou suspeita de desnutrição; e $C$, para gravemente desnutrido.
A Memorial Symptom Assessment Scale (MSAS), adaptada para a população brasileira por um grupo de especialistas em oncologia e epidemiologia ${ }^{10}$, foi aplicada para avaliar os sintomas que mais prevalecem nesses indivíduos. $O$ instrumento citado proporciona a combinação de sintomas físicos e psicológicos com seus respectivos graus de gravidade, frequência e grau de aflição, provocado pelos sintomas, por meio de uma escala de pontos do tipo Likert. Trata-se de um instrumento de autorrelato, no qual os pacientes atribuem um valor numérico de 1 a 4 pontos para a frequência e intensidade dos sintomas, bem como de 0 a 4 para o grau de incômodo experimentado durante a última semana. Cabe ressaltar que a ordenação das respostas revela que uma maior pontuação indica pior quadro clínico. Adicionalmente, a partir dos dados coletados, são calculadas 5 subescalas: PHYS-H, sintomas físicos de alta prevalência; PHYS-L, sintomas físicos de baixa prevalência; PSYCH, sintomas psicológicos; GDI, escala global de sofrimento e o TMSAS, escore total da escala de avaliação de sintomas, que abrange todos os itens do MSAS ${ }^{11}$.

Após análise dos dados da avaliação antropométrica, os pacientes foram classificados de acordo com o estado nutricional, levando em consideração os resultados da ASG-PPP.

Para a análise dos dados, no Statistical Package for Social Science ${ }^{\circledR}$ (SPSS), versão 22.0, foram realizados os cálculos da média, desvio padrão e a frequência absoluta (n) e relativa (\%); já para as comparações entre as variáveis, foi utilizado o teste análise de variância (ANOVA), considerando um nível de significância estatística de 95\%.

A presente pesquisa foi aprovada pelo Comitê de Ética em Pesquisa ( $n^{\circ} 2.841 .531$ ) do Centro Universitário Estácio de Sergipe. Foram incluídos apenas pacientes que, além de preencherem os critérios de inclusão, também assinaram o Termo de Consentimento Livre e Esclarecido (TCLE).

\section{RESULTADOS}

Foram avaliados 52 pacientes, com idade média de $59,62 \pm 15,51$ anos, sendo $53,85 \%$ do sexo masculino. Do total de participantes, $82,69 \%$ estavam acometidos por algum tipo de neoplasia. Desse grupo, cerca de 41,86\% estavam moderadamente desnutridos e $27,91 \%$, gravemente desnutridos (Tabela 1).

Vale ressaltar que são escassas, na literatura, pesquisas que associaram os resultados da MSAS ao estado nutricional, conforme observado no presente estudo. Porém, é possível verificar correlações entre o MSAS com sexo, etnia, estado civil e localização do tumor dos pacientes ${ }^{11}$.

Entre os 32 sintomas avaliados, os mais frequentes foram: "eu não pareço mais eu mesmo" (78,85\%), "perda de 
peso" (73,07\%), "dor" (69,23\%), "tristeza" (65,38\%), "boca seca" (57,69\%) e "falta de energia" (55,76\%). Na escala de frequência, prevaleceram os graus 3 e 4 . Em relação à escala de intensidade, houve predomínio dos graus 2, 3 e 4, o incômodo, por sua vez, prevaleceu com os números 3 e 4, de modo geral (Tabela 2).
Tabela 1 - Classificação do estado nutricional dos pacientes oncológicos de acordo com o ASG- PPP, em Hospital de Urgência em Aracaju, SE, 2018.

\begin{tabular}{lcc}
\hline Variáveis & $\mathbf{n = 5 2}$ & $\%$ \\
\hline Bem nutrido & 13 & 30,23 \\
Moderadamente desnutrido & 18 & 41,86 \\
Gravemente desnutrido & 12 & 27,91 \\
\hline
\end{tabular}

Tabela 2 - Avaliação dos sintomas mais prevalentes de acordo com a escala de frequência, intensidade e incômodo dos pacientes internados em um Hospital de Urgência em Aracaju, SE, 2018.

\begin{tabular}{|c|c|c|c|c|c|c|c|c|c|c|c|c|c|c|}
\hline \multirow[t]{2}{*}{ Sintomas } & \multicolumn{4}{|c|}{ Frequência } & \multicolumn{5}{|c|}{ Intensidade } & \multicolumn{5}{|c|}{ Incômodo } \\
\hline & $\%$ & 1 & 2 & 3 & 4 & 1 & 2 & 3 & 4 & 0 & 1 & 2 & 3 & 4 \\
\hline $\begin{array}{l}\text { 1-Dificuldade para } \\
\text { se concentrar }\end{array}$ & 32,69 & --- & 52,94 & 23,53 & 23,53 & 5,89 & 23,52 & 47,07 & 23,52 & 11,75 & 5,88 & 17,68 & 52,95 & 11,75 \\
\hline 2-Dor & 69,23 & --- & 27,78 & 36,11 & 36,11 & 2,77 & 50 & 13,88 & 33,33 & 5,55 & 2,78 & 5,55 & 30,56 & 55,56 \\
\hline 3-Falta de energia & 55,76 & 3,4 & 13,80 & 41,38 & 41,38 & 10,35 & 44,83 & 24,13 & 20,69 & --- & 10,34 & 10,35 & 27,59 & 51,72 \\
\hline 4-Tosse & 48,07 & 4 & 28 & 32 & 36 & 20 & 52 & 4 & 24 & 4 & 9,16 & 4 & 40 & 36 \\
\hline 5-Nervosismo & 46,15 & 4,17 & 37,5 & 33,33 & 25 & 25 & 41,67 & 16,67 & 16,67 & 20,83 & 20,83 & 16,67 & 16,67 & 25 \\
\hline 6-Boca seca & 57,69 & ---- & 33,33 & 36,66 & 30 & 13,33 & 56,67 & 13,33 & 16,66 & --- & 30 & 6,67 & 30 & 33,33 \\
\hline 7-Enjoo & 36,53 & --- & 36,84 & 36,84 & 26,32 & 15,78 & 57,89 & 15,78 & 10,53 & 5,27 & 15,78 & 5,27 & 36,84 & 36,84 \\
\hline 8-Sonolência & 44,23 & 4,35 & 21,7 & 43,47 & 30,43 & 21,74 & 56,52 & --- & 21,74 & ---- & 39,13 & 4,35 & 39,13 & 17,39 \\
\hline $\begin{array}{l}\text { 9-Dormência ou } \\
\text { formigamento nas } \\
\text { mãos/pés }\end{array}$ & 44,23 & 4,35 & 17,39 & 39,13 & 39,13 & 13,04 & 52,17 & 21,74 & 13,04 & ---- & 13,04 & 8,70 & 34,78 & 43,47 \\
\hline $\begin{array}{l}\text { 10-Dificuldade } \\
\text { para dormir }\end{array}$ & 48,07 & ---- & 12 & 48 & 40 & 32 & 36 & 4 & 28 & 12 & 12 & 15 & 12 & 48 \\
\hline 11-Empazinado & 44,23 & ---- & 26,09 & 34,78 & 36 & 26,09 & 39,13 & 4,43 & 30,43 & 4,43 & 13,04 & 17,39 & 13,04 & 52,17 \\
\hline $\begin{array}{l}\text { 12-Problema para } \\
\text { urinar }\end{array}$ & 28,84 & 6,67 & 20 & 26,67 & 46,67 & 13,33 & 33,33 & 20 & 33,33 & ---- & 13,33 & 6,67 & 33,33 & 46,67 \\
\hline 13-Vômitos & 34,61 & ---- & 44,44 & 33,33 & 22,22 & 11,11 & 50 & 22,22 & 16,67 & ---- & 11,11 & 5,55 & 44,44 & 38,89 \\
\hline 14-Falta de ar & 42,30 & ---- & 50 & 27,28 & 22,73 & 13,63 & 40,91 & 31,82 & 13,64 & ---- & 9,09 & ---- & 36,36 & 54,55 \\
\hline 15-Diarreia & 9,61 & ---- & 100 & ---- & $\begin{array}{ll}--- \\
\end{array}$ & 20 & ---- & 80 & --- & ---- & 20 & --- & 40 & 40 \\
\hline 16-Tristeza & 65,38 & ---- & 35,30 & 32,35 & 32,35 & 11,77 & 58,82 & 11,77 & 17,65 & ---- & 8,82 & 8,82 & 35,30 & 47,05 \\
\hline 17-Suor & 34,61 & 5,56 & 50 & 38,89 & 5,55 & 33,33 & 55,55 & 5,55 & ---- & 5,55 & 11,11 & 22,22 & 50 & 11,11 \\
\hline 18-Preocupações & 48,07 & ---- & 48 & 40 & 12 & 28 & 56 & 8 & 8 & 4 & 8 & 16 & 44 & 28 \\
\hline $\begin{array}{l}\text { 19-Problemas } \\
\text { com desejo ou } \\
\text { atividade sexual }\end{array}$ & ---- & ---- & ---- & ---- & ---- & ---- & --- & ---- & ---- & ---- & ---- & ---- & ---- & ---- \\
\hline 20-Coceira & 23,07 & 33,33 & ----- & 66,66 & ----- & 41,67 & 58,33 & ----- & ----- & ----- & 25 & 8,33 & 33,33 & 33,33 \\
\hline 21-Falta de apetite & 36,53 & ---- & 31,58 & 21,05 & 47,36 & ----- & 63,16 & ----- & 36,84 & ----- & ----- & 15,79 & 42,10 & 42,10 \\
\hline 22-Tontura & 42,30 & ---- & 54,55 & 31,81 & 13,63 & 36,36 & 31,82 & 22,73 & 9,09 & 4,55 & 9,09 & 22,73 & 36,36 & 27,27 \\
\hline $\begin{array}{l}\text { 23-Dificuldade } \\
\text { para engolir }\end{array}$ & 32,69 & ---- & 5,88 & 41,17 & 52,94 & 5,88 & 41,17 & 5,88 & 47,06 & ---- & 11,76 & ---- & 47,06 & 41,17 \\
\hline 24-Irritado & 38,46 & --- & 20 & 35 & 35 & 20 & 40 & 25 & 10 & ---- & 25 & 10 & 35 & 25 \\
\hline 25-Feridas na boca & & & & & 11,53 & 50 & 50 & ----- & ----- & ----- & 66,67 & 33,33 & ----- & ---- \\
\hline 26-Mudança no gos & sto dos & imentos & & & 26,92 & 21,43 & 42,86 & 14,28 & 21,43 & ----- & 35,72 & 28,57 & 7,14 & 28,57 \\
\hline 27-Perda de peso & & & & & 73,07 & 5,26 & 50 & 34,21 & 10,53 & 7,90 & 23,68 & 15,79 & 28,95 & 23,68 \\
\hline 28-Perda de cabelo & & & & & 21,15 & 18,18 & 9,09 & 27,27 & 45,46 & ----- & 9,09 & 18,18 & 27,27 & 45,46 \\
\hline 29-Prisão de ventre & & & & & 53,84 & 3,57 & 60,71 & 10,72 & 25 & ----- & 14,29 & 14,29 & 39,28 & 32,14 \\
\hline 30-Inchaço nos bra & ços ou & ernas & & & 30,76 & ----- & 43,75 & 37,5 & 18,75 & ----- & 12,5 & ---- & 50 & 37,5 \\
\hline 31-Eu não pareço $\mathrm{m}$ & nais eu & smo (a) & & & 78,85 & 17,07 & 58,54 & 19,51 & 4,88 & 2,44 & 34,15 & 26,83 & 31,70 & 4,88 \\
\hline 32-Alterações na pe & & & & & 36,53 & 15,79 & 47,37 & 15,79 & 21,05 & ----- & 26,31 & 10,53 & 47,37 & 15,79 \\
\hline
\end{tabular}

Frequência: Raramente (1); Às vezes (2); Frequentemente (3); Quase o tempo todo (4).

Intensidade: Leve (1); Moderada (2); Grave (3); Muito grave (4).

Incômodo: Nada (0); Um pouco (1); Mais ou menos (2); Consideravelmente (3); Muito (4). 
Com relação às subescalas do MSAS, GDI $(1,5 \pm 0,9)$ e PHYS-H $(1,31 \pm 0,84)$ destacaram-se em comparação com as demais (Tabela 3). Além disso, foi observada uma associação estaticamente significativa entre os sintomas físicos (PHYS-H e PHYS-L) e TMSAS com o estado nutricional dos pacientes (Tabela 4) $(p<0,05)$.

\begin{tabular}{|c|c|c|c|c|c|c|c|c|c|}
\hline \multicolumn{10}{|c|}{ Tabela 3 - Média e desvio padrão das subescalas do MSAS. } \\
\hline \multicolumn{2}{|c|}{ PHYS-H } & \multicolumn{2}{|c|}{ PHYS-L } & \multicolumn{2}{|c|}{ PSYCH } & \multicolumn{2}{|c|}{ GDI } & \multicolumn{2}{|c|}{ TMSAS } \\
\hline Média & $\pm \mathrm{DP}$ & Média & $\pm \mathrm{DP}$ & Média & $\pm \mathrm{DP}$ & Média & $\pm \mathrm{DP}$ & Média & $\pm \mathrm{DP}$ \\
\hline 1,31 & 0,84 & 0,89 & 0,58 & 1,24 & 0,86 & 1,5 & 0,9 & 1,12 & 0,61 \\
\hline
\end{tabular}

PHYS-H = Subescala de sintomas físicos de alta prevalência; PHYS-L = Subescala de sintoma físicos de baixa prevalência; PSYCH = Subescala de sintomas psicológicos; GDI = Subescala global de sofrimento; TMSAS = Subsescala geral do MSAS.

Tabela 4 - Associação do estado nutricional com as subescalas do MSAS.

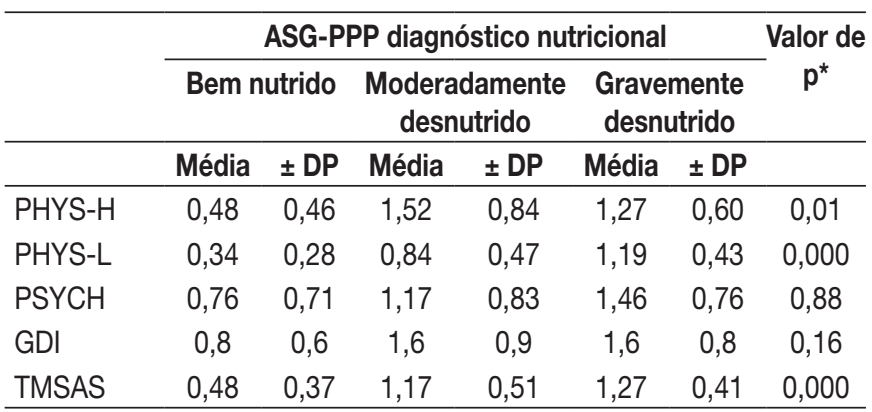

PHYS-H = Subescala de sintomas físicos de alta prevalência; PHYS-L = Subescala de sintomas físicos de baixa prevalência; $\mathrm{PHYCH}=$ Subescala de sintomas psicológicos; GDI = Subescala global de sofrimento; TMSAS = Subescala geral do MSAS. ANOVA $p<0,05$.

\section{DISCUSSÃO}

A amostra apresentou alto índice de desnutrição. No Inquérito Brasileiro de Avaliação Nutricional Hospitalar (IBRANUTRI) ${ }^{8}$, que avaliou 4 mil pacientes internados na rede pública hospitalar de vários estados brasileiros, a prevalência de desnutrição foi de $48,1 \%$, sendo que $12,6 \%$ apresentavam desnutrição grave e $35,5 \%$, moderada. Segundo o mesmo estudo, a explicação para esse achado se deve a um conjunto de condições encontradas no ambiente hospitalar, podendo ter causas relacionadas ao próprio paciente, como tipo e extensão da doença primária, ingesta alimentar ou suporte nutricional inadequado.

A ASG-PPP é amplamente utilizada em pacientes oncológicos hospitalizados e apresenta sensibilidade de $98 \%$ e especificidade de $82 \%$. A desnutrição nesses indivíduos é causada por vários fatores, podendo estar relacionados à presença do tumor, que ocasiona diversas alterações metabólicas, ou mesmo ao tratamento e seus efeitos colaterais, que aumenta a morbimortalidade ${ }^{12,13}$. Essas alterações metabólicas estão relacionadas com os substratos de energia, acarretando aumento da lipólise, metabolismo glicídico, marcadores inflamatórios e substâncias catabólicas, com decorrente elevação do gasto energético ${ }^{14}$.

Em um estudo realizado por Heredia et al. ${ }^{14}$, no qual a ASG-PPP foi aplicada em 33 pacientes oncológicos, a prevalência de pacientes com necessidade de intervenção nutricional foi de $42,4 \%$. O resultado foi semeIhante ao presente estudo, que apresentou um índice de $41,86 \%$ em relação aos pacientes moderadamente desnutridos.

Nos cuidados paliativos, os pacientes apresentam os sintomas em concomitância com outros, e devem ser investigados por instrumentos específicos. Assim, é de suma importância a identificação destes por parte dos profissionais de saúde, desde o momento da internação até a alta hospitalar, com o objetivo de direcionar o cuidado e promover qualidade de vida ${ }^{13,15,16}$.

De acordo com Costa e Othero ${ }^{15}$, em cuidados paliativos, a prevenção e o controle dos sintomas são um desafio para a assistência interdisciplinar e multiprofissional. Mesmo existindo especificidades de cada área profissional, todos que atuam nos casos referidos devem ser capazes de identificar sintomas e conhecer técnicas básicas de manejo e seus encaminhamentos, de modo a minimizar a gravidade, frequência, duração e intensidade desses.

Os resultados demonstram elevada prevalência de sintomas físicos e psicológicos, em virtude desta circunstância, tornando-se indispensável a adoção de uma estratégia terapêutica mais eficiente ${ }^{17}$. Neste contexto, uma intervenção nutricional eficiente auxilia o paciente a lidar com os sintomas decorrentes do estágio da doença, na busca de estratégias para minimizar os sintomas e agravos ${ }^{18}$.

Diante do exposto, vale ressaltar a importância da avaliação nutricional, diagnóstico e acompanhamento individualizado, com objetivo de melhorar a relação com o alimento, ajudar no controle dos sintomas, preservar o peso e a composição corporal, melhorando, desse modo, a assistência nutricional e a resposta imunológica ${ }^{19}$.

\section{CONCLUSÃO}

Conclui-se que a progressão da doença terminal envolve sintomas físicos, psicológicos, espirituais e sociais. Esses, por sua vez, não resultam apenas da progressão da doença primária, mas também do tratamento e comorbidades associadas, o que se associa ao risco nutricional, tornando evidente que a identificação precoce, a avaliação e o tratamento dos sintomas são de importância fundamental em cuidados paliativos. Os resultados apontam para a necessidade de mais estudos na temática, a fim de otimizar demandas e intervenções. 


\section{REFERÊNCIAS}

1. Sawatzky R, Porterfiel P, Lee J, Dixon D, Lounsbury K, Pesut B, et al. Conceptual foundations of a palliative approach: a knowledge synthesis. BMC Palliative Care. 2016;15(1):5.

2. World Health Organization (WHO). Palliative care. Geneva: World Health Organization; 2018. [cited 2018 Apr 03]. Available from: http://www.who.int/mediacentre/factsheets/fs402/ en/

3. Oliveira LC. Cuidados paliativos: por que precisamos falar sobre isso? Rev Bras Cancerol. 2019;65(4):e-04558.

4. Victor GHGG. Cuidados paliativos no mundo. Rev Bras Cancerol. 2016;62(3):267-70.

5. Correia FR, De Carlo MMRP. Avaliação de qualidade de vida no contexto dos cuidados paliativos: revisão integrada de literatura. Rev Latino-Am Enfermagem. 2012;20(2):401-10.

6. Blauwhoff-Buskermolen S, Ruijgrok C, Ostelo RW, de Vet HCW, Verheul HMW, van der Schueren MAE, et al. The assessment of anorexia in patients with cancer: cut-off values for the FAACT-A/CS and the VAS for appetite. Support Care Cancer. 2016;24(2):661-6.

7. Reim D, Friess H. Feeding challenges in patients with esophageal and gastroesophageal cancers. Gastrointest Tumors. 2016;2(4):166-77.

8. Waitzberg DL, Caiaffa WT, Correia MI. Hospital malnutrition: the Brazilian national survey (IBRANUTRI): a study of 4000 patients. Nutrition. 2001;17(7-8):573-80.

9. Gonzalez MC, Borges LR, Silveira DH, Assunção MCF, Orlandi SP. Validação da versão em português da avaliação subjetiva global produzida pelo paciente. Rev Bras Nutr Clin. 2010;25(2):102-8.
10. Chang VT, Hwang SS, Feuerman M, Kasimis BS, Thaler HT. The Memorial Symptom Assessment Scale Short Form (MSAS-SF). Cancer. 2000;89(5):1162-71.

11. Souza ALLP, Martins TCF, Pedrosa TM, Silva DGF, Mello MRSP, Muzi CD, et al. Global and multidimensional symptom assessments in patients presenting abdominal neoplasms. Aquichan. 2019;19(1):16-20.

12. Miranda TV, Neves FMG, Costa GNR, Souza MAM. Estado nutricional e qualidade de vida de pacientes em tratamento quimioterápico. Rev Bras Cancerol. 2013;59(1):57-64.

13. Cuppari L. Nutrição clínica no adulto. $3^{\mathrm{a}}$ ed. Barueri: Manole; 2014.

14. Heredia M, Canales S, SáezC, Testillano M. The nutritional status of patients with colorectal cancer undergoing chemotherapy. Farm Hosp. 2008;32(1):35-7.

15. Costa APP, Othero MB. Conceitos, princípios e formação em cuidados paliativos. In: Reabilitação em cuidados paliativos. Loures: Lusodidacta; 2014. p.23-36.

16. Menezes JR, Luvisaro BMO, Rodrigues CF, Muzi CD, Guimarães RM. Confiabilidade teste-reteste da versão Brasileira do instrumento Memorial Symptom Assessment Scale para avaliação de sintomas em pacientes oncológicos. Einstein. 2017;15(2):148-54.

17. Dalal S, Del Fabbro E, Bruera E. Symptom control in palliative care - Part I: oncology as a paradigmatic example. J Palliat Med. 2006;9(2):391-408.

18. O'Hara PD. The management of nutrition for palliative care patients. Health Social Care. 2017;2(1):21-38.

19. Rebouças LM, Callegaro E, Gil GOB, Silva MLG, Maia MAC, Salvajoli JV. Impacto da nutrição enteral na toxicidade aguda e na continuidade do tratamento dos pacientes com tumores de cabeça e pescoço submetidos a radioterapia com intensidade modulada. Radiol Bras. 2011;44(1):42-6.

Local de realização do estudo: Hospital de Urgência de Sergipe, Aracaju, SE, Brasil.

Conflito de interesse: Os autores declaram não haver.

Apresentado no IV Congresso Nacional de Alimentos e Nutrição e I Congresso de Pós-Graduação em Alimentação e Nutrição, Ouro Preto, MG, 31 de maio de 2019. 